Original article

\title{
The effect of the Suicide Prevention Program (SPP) on the characteristics of Israeli soldiers who died by suicide after its implementation
}

\author{
Leah Shelef ${ }^{\mathrm{a}, \mathrm{d}}$, Ishai Nir ${ }^{\mathrm{a}, \mathrm{d}, *}$, Lucian Tatsa-Laur ${ }^{\mathrm{a}, \mathrm{d}}$, Ron Kedem ${ }^{\mathrm{b}, \mathrm{d}}$, Niv Gold ${ }^{\mathrm{a}, \mathrm{d}}$, \\ Tarif Bader ${ }^{\mathrm{c}, \mathrm{d}}$, Ariel Ben Yehuda ${ }^{\mathrm{a}, \mathrm{d}}$ \\ a Mental Health Department, Israel Defense Force Medical Corps, Ramat Gan, Israel \\ b Statistican, Medical Corps- Israel Defense Forces, Ramat Gan, Israel \\ ' Surgeon General's Headquarters, Israel Defense Force, Ramat Gan, Israel \\ ${ }^{\mathrm{d}}$ Department of Military Medicine, Hebrew University, Jerusalem, Israel
}

\section{A R T I C L E I N F O}

\section{Article history:}

Received 15 June 2019

Received in revised form 18 August 2019

Accepted 26 August 2019

Available online 21 September 2019

Keywords:

Suicide prevention

Suicide rate

Israeli Defense Force

Risk factors

\begin{abstract}
A B S T R A C T
Background: Suicide is major cause of death in the IDF. The Suicide Prevention Program (SPP) led to significant reduction in yearly rates of suicide. A study regarding demographic changes of those who died by suicide was done to further investigate its affect.

Method: Nested case control retrospective study based on medical and HR data gathered between 1992 and 2016. Participants were divided into four groups: soldiers who died by suicide and non-suicidal soldiers, before and after SPP implementation.

Results: Multivariate analysis with suicide as the binary logistic dependent variable before and after implementation of the SPP among four groups revealed that before SPP the OR was higher for males (OR, 7.885; 95\% CI, 5.071-12.259; $>0.001)$ compared to after (OR, 3.281; 95\% CI, 1.600-6.726; $p=0.001)$. For support unit soldiers the values before SPP were OR, 14.962 and 95\% CI, 8.427-26.563 $(p<0.001)$ while after SPP they were OR, 6.304 and 95\% CI, 3.334-11.919 $(p<0.001)$. After SPP, OR was higher for psychiatric diagnosis at recruitment (OR, 5.830; 95\% CI, 2.046-16.612; $p=0.001)$ than before SPP $(\mathrm{OR}, 2.422 ; 95 \% \mathrm{CI}$ 1.526-3.842; $p<0.001)$.For soldiers from Ethiopian ethnicity, after SPP values were higher (OR, 8.130 and 95\% CI, 2.868-23.047 ( $p<0.001$ ) compared to before(OR, 3.522; 95\% CI, 1.2891-6.650; $p<0.001$ ). For those of Druse religion before values (OR, 4.027; 95\% CI, 2.211-7.331; $p<0.001$ ) were significant but not after. Conclusions: While the SPP succeeded in reducing risk of suicide in situational factors, dispositional risk factors were not affected by the SPP. The OR decreased in critical masses and rose in unique and smaller groups.
\end{abstract}

(C) 2019 Elsevier Masson SAS. All rights reserved.

\section{Introduction}

\subsection{Context}

Suicide is an important public health issue in the Israeli army [1] as it is in other military organizations worldwide. Between the years 1990 and 2016, excluding death as a result of military operational, suicide constitutes $41 \%$ of all fatalities among soldiers in compulsory military service [1].

The Suicide Prevention Program (SPP) that was introduced in 2006, included several components such as reducing weapon

\footnotetext{
${ }^{*}$ Corresponding author at: Mental Health Department, Israel Defense Force Medical Corps, Ramat Gan, Israel.

E-mail address: Ishai.nir@gmail.com (I. Nir).
}

accessibility, psychoeducation for soldiers and commanders and integration of mental health officers in fighting units [2,3]. Trend analysis showed lower suicide rates after intervention ( $\mathrm{HR}=0.44 ; 95 \%$ $\mathrm{CI}=0.34-0.56, \mathrm{p}<.001$ ), especially among males (Fig. 1 ).

A large study was conducted in 2017 in the IDF and its findings indicated that the factors that increase risk of death by suicide (DBS) are: male gender, of Ethiopian ethnicity, low socioeconomic status and having a low score in Adjustment Difficulties - a score given by a mental health officers following examination. In addition, Cluster B personality disorder (antisocial, borderline, histrionic, and narcissistic) and low to average motivation to serve in a combat unit where found to increase the risk for DBS in males only [4].

Some similarities were found between the IDF results and risk factors for DBS among U.S. military personnel. Those include gender and age i.e. young men [4-7], and mental illness [3,8]. 


\subsection{The present study}

In the current study the research question was whether there was a change in the characteristics of soldiers who died by suicide after the implementation of the SPP (2009-2016) in comparison to those who died by suicide before SPP implementation (19922008). The assumption was that before SPP implementation the risk factors for DBS were both dispositional (gender, cognitive level, socio-economic status, mental illness etc.) and situational (serving in combat unit), while after SPP implementation and the resulting reduction in the rate of DBS, dispositional factors would prove to be more dominant.

\section{Methods}

\subsection{Design and settings}

Nested case control (NCC) study in which cases and controls are drawn from the population in a fully enumerated cohort [9].

The study was based on data from HR and from medical records, gathered between 5/1/1992 and 31/12/2016. The personal and medical records were divided into two groups. The first included soldiers that were drafted into military service during the years 1992 to 2006. The second group included the records of soldiers drafted between 2009 and 2016. Soldiers drafted during the years 2007 to 2008 were excluded since during those years the SPP gradual implementation took place and as a result soldiers' exposure to the SPP varied and could not be regarded on par with those drafted after full implementation had been completed [3].

\subsection{Bias}

Selection and information bias were minimized by selecting a population with similar characteristics (e.g., age-matched young people who passed qualifying military exams and were found suitable for military service) and using a well-defined procedure for determining outcome variables.

\subsection{Measurements}

\subsubsection{Study participants}

The potential study population consisted of 1,526,853 active duty mandatory service IDF soldiers that served during the years 1992-2016. Of those, 17 soldiers who were drafted into professional rather than mandatory service were excluded, leaving $1,526,836$ records. Soldiers recruited during the implementation of the SPP and prior to its full completion (2007-2008) were also excluded, leaving a cohort of 1,462,882 from whose records was derived the data for this study. Participants were divided into four groups: soldiers who died by suicide and non-suicidal soldiers, before and after SPP implementation. The number of DBSs in the study cohort during those years was found to consist of 525 cases. The normal length of mandatory service in the IDF is 1100 days for males and 735 days for females. In order to establish a numerator of DBSs occurring during mandatory service all DBSs of male soldiers who served more than 1100 days and female soldiers who served more than 735 days at the time of suicide were excluded, as were soldiers who entered professional service earlier than the usual time, for whatever reasons. This reduced the group of DBSs in the aforementioned years to 491 (Table 1 ).

\subsubsection{Independent variables}

Ethnicity. Data regarding ethnicity was retrieved from the IDF's HR database. This variable was categorized into seven groups: Israel, Former Soviet Union (FSU), Ethiopia, Africa, Asia, Western countries and Australia-New Zealand. It is important to notice that this variable referred to ethnicity in the broad sense as many of the soldiers were born in Israel but were categorized based on their ancestors' country of origin.

Socioeconomic status (SES). SES was determined using location of residence as a proxy variable and basing it on the 10-point scale created by the Israeli Central Bureau of Statistics (ICBS). The ICBS divided the country geographically into residential clusters, and assigned them numbers on a scale from 1 to 10 , with a higher number reflecting higher SES. Each soldier was assigned a status according to the number assigned to his residential area, as follows: low (1-4), average (5-7), and high (8-10).

Intelligence score. Cognitive functioning was measured by the Intellectual Rating Score (IRS) [10,11]. The total IRS score is regarded as being equivalent to a normally distributed IQ score. Scored on a 9-point scale running from 10 to 90 with 10 -point increments, a score of $10-30$ is considered low, $40-60$ is average and 70-90 is high.

Combat duty. For the current study, we separated the soldiers into three groups: Combat soldiers, combat support soldiers who, while also carrying firearms are not expected to engage in direct combat and soldiers in back units who do not carry firearms and are not expected to engage in combat.

Religion. The soldiers' religion was retrieved from the HR database. The three groups relevant to the current study were Jews, Druse and others (who were comprised mainly of former Soviet Union immigrants not classified as Jewish).

\subsubsection{Dependent variable \\ Soldiers who died by suicide.}

\subsection{Statistical analysis}

Analyses were performed with IBM SPSS Statistics for Windows, version 23.0. Armonk, NY: IBM Corp. Tests significant level was $\alpha=0.05$. Models included gender, socio-economic status, ethnicity, religion, and intellect rating score, pre-induction mental health diagnosis, and combat unit. Univariate analysis with a minimal model were adjusted for gender and IQ. Multivariate analysis with suicide binary logistic dependent variable before (1992-2008) and after (2009-2016) implementation of the SPP.

\subsection{Ethics approval}

The Institutional Review Board of the IDF Medical Corps approved the study and waived the requirement for informed consent on condition of preserving participants' anonymity.

\section{Results}

\subsection{Characteristics}

Characteristics of the study population before and after SPP sorted by DBS and no-DBS are presented in Table 1 . Final cohort characteristics (Table 1 ) will be described by categories.

Gender. Comparing the DBS rates in the cohort before and after SPP implementation it was found that the proportion of males was significantly higher $(p<0.001)$ before SPP $(91.7 \% v s .86 .8 \%)$, while the proportion of females was higher after SPP (13.2\% vs. $8.3 \%)$.

Intelligence score. The proportion of soldiers with a high intellectual rating score was higher among those who committed DBS (35.3\% vs. $26.3 \%$; p < 0.001 ), while soldiers with a lower and average intellectual rating score were more prevalent among noDBS (32.7\% and $41.0 \%$ vs. $28.5 \%$ and $36.2 \%$ respectively).

Type of service. The proportion of DBSs among soldiers serving in combat units or in back units was higher before SPP 
Table 1

Characteristics of the study population before and after SPP $(\mathrm{N}=1,462,882)$.

\begin{tabular}{|c|c|c|c|c|c|c|c|c|c|}
\hline \multirow{2}{*}{$\begin{array}{l}\text { Implementation } \\
\text { of the SPP }\end{array}$} & \multirow[t]{2}{*}{ Variable } & & \multicolumn{2}{|c|}{ Non-Suicidal } & \multirow{2}{*}{$\begin{array}{l}\text { Suicide } \\
\mathrm{N}\end{array}$} & \multirow[b]{2}{*}{$\%$} & \multicolumn{2}{|l|}{ Total } & \multirow{2}{*}{$\begin{array}{l}P \\
\text { Exact Sig (2 sided) }\end{array}$} \\
\hline & & & $\mathrm{N}$ & $\%$ & & & $\mathrm{~N}$ & $\%$ & \\
\hline \multirow[t]{2}{*}{ \#Before } & Gender & Female & 427,987 & 43.6 & 35 & 8.3 & 428,022 & 43.6 & \\
\hline & & Male & 552,519 & 56.4 & 388 & 91.7 & 552,907 & 56.4 & $<0.001$ \\
\hline \multirow[t]{2}{*}{${ }^{\infty}$ After } & Gender & Female & 203,349 & 42.2 & 9 & 13.2 & 203,358 & 42.2 & \\
\hline & & Male & 278,536 & 57.8 & 59 & 86.8 & 278,595 & 57.8 & $<0.001$ \\
\hline \multirow[t]{3}{*}{ \# Before } & Inelegance score & Low & 318,285 & 32.7 & 119 & 28.5 & 318,404 & 32.7 & \\
\hline & & Average & 399,701 & 41.0 & 151 & 36.2 & 399,852 & 41.0 & \\
\hline & & High & 256,333 & 26.3 & 147 & 35.3 & 256,480 & 26.0 & $<0.001$ \\
\hline \multirow[t]{3}{*}{${ }^{\infty}$ After } & Inelegance score & Low & 173,955 & 36.3 & 20 & 29.4 & 173975 & 36.3 & \\
\hline & & Average & 186,577 & 38.9 & 28 & 41.2 & 186,605 & 38.9 & \\
\hline & & High & 118,714 & 24.8 & 20 & 29.4 & 118,734 & 24.8 & 0.457 \\
\hline \multirow[t]{3}{*}{ \#Before } & Type of service & Combat & 299,649 & 40.0 & 189 & 57.1 & 299,838 & 40.0 & \\
\hline & & Combat support & 2,323 & 0.3 & 13 & 3.9 & 2,336 & 0.3 & \\
\hline & & Back unit & 446,523 & 59.7 & 129 & 39.0 & 446,652 & 59.6 & $<0.001$ \\
\hline \multirow{3}{*}{${ }^{\infty}$ After } & Type of service & Combat & 157,396 & 32.7 & 32 & 47.1 & 157,428 & 32.7 & \\
\hline & & Combat support & 12,289 & 2.6 & 14 & 20.6 & 12,303 & 2.6 & \\
\hline & & Back unit & 312,196 & 64.8 & 22 & 32.4 & 312,218 & 64.8 & $<0.001$ \\
\hline \multirow[t]{2}{*}{${ }^{\#}$ Before } & ${ }^{\mu}$ Psychiatric Diagnosis & No & 971,445 & 99.1 & 416 & 98.3 & 971,861 & 99.1 & \\
\hline & & Yes & 9,061 & 0.9 & 7 & 1.7 & 9,068 & 0.9 & 0.120 \\
\hline \multirow[t]{2}{*}{${ }^{\infty}$ After } & ${ }^{\mu}$ Psychiatric Diagnosis & No & 473,361 & 98.2 & 64 & 94.1 & 473,425 & 98.2 & \\
\hline & & Yes & 8,524 & 1.8 & 4 & 5.9 & 8,528 & 1.8 & 0.033 \\
\hline \multirow[t]{7}{*}{ \#Before } & Origin Country & Australia-New Zealand & 7,377 & 0.8 & 3 & 0.7 & 7,380 & 0.8 & \\
\hline & & Western country & 253,825 & 26.0 & 109 & 26.0 & 253,934 & 26.0 & \\
\hline & & Asia + Oceania & 219,475 & 22.5 & 76 & 18.1 & 219,551 & 22.5 & \\
\hline & & Africa & 220,979 & 22.7 & 47 & 11.2 & 221,026 & 22.7 & \\
\hline & & Ethiopia & 17,614 & 1.8 & 26 & 6.2 & 17,640 & 1.8 & \\
\hline & & Former Soviet Union & 176,134 & 18.1 & 103 & 24.5 & 176,237 & 18.1 & \\
\hline & & Israel & 79,715 & 8.2 & 56 & 13.3 & 79,771 & 8.2 & $<0.001$ \\
\hline \multirow[t]{7}{*}{${ }^{\infty}$ After } & Origin Country & Australia-New Zealand & 2,500 & 0.5 & 2 & 2.9 & 2,502 & 0.5 & \\
\hline & & Western country & 82,942 & 17.3 & 13 & 19.1 & 82,955 & 17.3 & \\
\hline & & Asia + Oceania & 81,308 & 17.0 & 10 & 14.7 & 81,318 & 17.0 & \\
\hline & & Africa & 91,477 & 19.1 & 10 & 14.7 & 91,487 & 19.1 & \\
\hline & & Ethiopia & 17,253 & 3.6 & 7 & 10.3 & 17,260 & 3.6 & \\
\hline & & Former Soviet Union & 79,896 & 16.7 & 16 & 23.5 & 79,912 & 16.7 & \\
\hline & & Israel & 123,816 & 25.8 & 10 & 14.7 & 123,826 & 25.8 & 0.001 \\
\hline \multirow[t]{3}{*}{ \#Before } & Religion & Other & 5,658 & 0.6 & 4 & 0.9 & 5,662 & 0.6 & \\
\hline & & Druse & 16,496 & 1.7 & 31 & 7.1 & 16,527 & 1.7 & \\
\hline & & Jewish & 957,888 & 97.7 & 388 & 91.7 & 958,276 & 97.7 & $<0.001$ \\
\hline \multirow[t]{3}{*}{${ }^{\infty}$ After } & & Other & 36 & 0.0 & 0 & 0.0 & 36 & 0.0 & \\
\hline & & Druse & 10,051 & 2.1 & 1 & 1.5 & 10,052 & 2.1 & \\
\hline & & Jewish & 471,010 & 97.9 & 67 & 98.5 & 471,077 & 97.9 & 0.936 \\
\hline \multirow[t]{3}{*}{ \#Before } & ${ }^{\sharp} \mathrm{SES}$ & Low & 27,336 & 2.9 & 22 & 5.4 & 27,358 & 2.9 & \\
\hline & & Average & 479,902 & 50.3 & 213 & 52.6 & 480,115 & 50.3 & \\
\hline & & High & 447,666 & 46.9 & 170 & 42.0 & 447,836 & 46.9 & 0.003 \\
\hline${ }^{\infty}$ After & ${ }^{¥} \mathrm{SES}$ & Low & 17,019 & 3.6 & 4 & 5.9 & 17,023 & 3.6 & \\
\hline & & Average & 245,710 & 51.3 & 39 & 57.4 & 245,749 & 51.3 & \\
\hline & & High & 216,143 & 45.1 & 25 & 36.8 & 216,168 & 45.1 & 0.276 \\
\hline
\end{tabular}

Note: \#Before: 1992-2008; $\infty$ After: 2009-2016; ¥SES-Socioeconomic status.

implementation than after implementation ( $57.1 \%$ vs. $47.1 \%$ among combat units and $39.0 \%$ vs. 32.4 among back units). In contrast, among soldiers from combat support units, the proportion of DBS was higher after SPP implementation than before (20.6\% vs. 3.9\%).

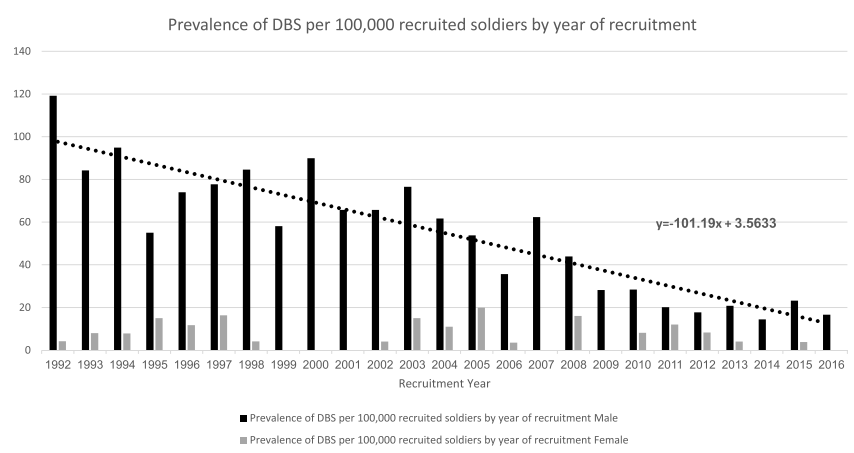

Fig. 1. Prevalence of DBS per 100,000 recruited soldiers by year of recruitment.
Psychiatric diagnosis on induction day. A minority of the subjects who committed DBS had a psychiatric diagnosis on induction day ( $1.7 \%$ vs. $98.3 \%$ before; $5.9 \%$ vs. $94.1 \%$ after). As can be seen, among the soldiers who died by suicide the proportion of those who had psychiatric diagnosis was higher after SPP than before (5.9\% vs. $1.7 \% ; p=0.003)$.

Ethnicity. Among the soldiers who committed DBS, the proportion of those from Western countries' or Asian ethnicity showed a marked decline after SPP implementation (Western countries-26.0\% vs. 19.1\%, Asian countries $18.1 \%$ vs. 14.7\%). Among those who were ethnically from the Former Soviet Union there was a mild decline in the proportion of DBS following the SPP $(24.5 \% v s$. 23.5\%). In contrast, the DBS proportion of soldiers from AustraliaNew Zealand ethnicity increased after SPP implementation $(0.7 \%$ vs. $2.9 \%$ ), as did the DBS proportions of those of Ethiopian ethnicity (6.2\% vs. $10.3 \%$ ) and ethnic Israelis (13.3\% before, vs. $14.7 \%$ after).

Religion. Among those of Jewish religion the proportion of DBS was higher after SPP than before (91.7\% vs. 97.9\%), while there was a decline following the SPP among Druse (7.1\% vs. 1.5\%) and those of other religions ( $0.9 \%$ vs. $0.0 \%)$, 
Socioeconomic status (SES). Of the total number of DBS amongst the soldiers, the proportion of those who came from low or average SES, was higher after SPP implementation than before (5.4\% vs. $5.9 \%$; $52.6 \%$ vs. $57.4 \%$ ), namely, the proportion of soldiers from high SES decreased (42.0\% before, $36.8 \%$ after).

Table 2 shows the changes in two variables that characterize soldiers who DBS before and after the SPP implementation. While the proportion of combat support soldiers among the DBS group was higher after SPP implementation than before (20.6\% vs. 3.9\%), the proportion of combat soldiers decrease after SPP, as compared to before ( $47.1 \% v s .57 .1 \%)$, as did the proportion of soldiers serving in back units $(39.0 \% v s .37 .8 ; p<0.001)$. The proportion of soldiers with psychiatric diagnosis, on the other hand, increased from $1.7 \%$ prior to SPP implementation, to $4.7 \%$ following it ( $p=0.029)$.

Table 3 presents the results of a univariate analysis with a minimal model, adjusted for gender and IQ, which was performed in order to calculate odds ratio (OR). As can be seen, prior to SPP implementation, the risk for DBS was more significant for those of male gender $(\mathrm{OR}=8.45 ; 95 \% \mathrm{CI}, 5.983-11.958 ; p<0.001)$. After SPP implementation the risk for male gender was still significant $(p=0.001)$, however, the OR for male gender decreased from 8.459 to 4.746 .

Before the SPP, a high intelligence score constituted a risk factor for DBS (OR $=1.010 ; 95 \% \mathrm{CI}, 1.005-1.016 ; p=0.001)$. Comparing the mean level of intelligence of both soldiers who committed DBS and soldiers who did not, prior to and after the implementation of the SPP, the findings revealed that for those who did not commit DBS the mean level of intelligence was significantly lower after SPP (before: $\mu=110.22, \quad \mathrm{SD}=15.418$; after: $\mu=109.32 \mathrm{SD}=15.533$; $p<0.001$ ).

In general, the average intelligence level of those who committed DBS was higher compared to the average intelligence level of those who did not, both before (113.15 vs. 110.22) and after (112.87 vs. 109.32) the SPP. For those who committed DBS, the average intelligence score after the SPP was also lower but the difference did not reach statistical significance (113.15 vs. 112.87; $p=0.898$ ).

Service in combat units or combat support units constitutes a significant risk factor for DBS, both before and after the SPP. The risk for combat support soldiers is much higher, but the risk level

Table 2

Characteristics of suicide and non-suicidal soldiers $(\mathrm{N}=1,462,882)$.

\begin{tabular}{|c|c|c|c|c|c|c|c|c|c|}
\hline & \multirow[t]{2}{*}{ Variable } & & \multicolumn{2}{|l|}{${ }^{\#}$ Before } & \multicolumn{2}{|l|}{${ }^{\infty}$ After } & \multicolumn{2}{|l|}{ Total } & \multirow[t]{2}{*}{$\begin{array}{l}P \\
\text { Exact Sig (2 sided) }\end{array}$} \\
\hline & & & $\mathrm{N}$ & $\%$ & $\mathrm{~N}$ & $\%$ & $\mathrm{~N}$ & $\%$ & \\
\hline \multirow[t]{2}{*}{ Non suicidal } & Gender & Female & 427,987 & 43.6 & 203,349 & 42.2 & 631,336 & 43.2 & \\
\hline & & Male & 552,519 & 56.4 & 278,536 & 57.8 & 831,055 & 56.8 & $<0.001$ \\
\hline \multirow[t]{2}{*}{ Suicide } & Gender & Female & 35 & 8.3 & 9 & 13.2 & 44 & 9.0 & \\
\hline & & Male & 388 & 91.7 & 59 & 86.8 & 447 & 91.0 & 0.184 \\
\hline \multirow[t]{3}{*}{ Non suicidal } & Inelegance Score & Low & 318,285 & 32.7 & 173,955 & 36.3 & 492,240 & 33.9 & \\
\hline & & Average & 399,701 & 41.0 & 186,577 & 38.9 & 586,278 & 40.3 & \\
\hline & & High & 256,333 & 26.3 & 118,714 & 28.8 & 375,047 & 25.8 & $<0.001$ \\
\hline \multirow{3}{*}{ Suicide } & Inelegance Score & Low & 119 & 28.5 & 20 & 29.4 & 139 & 28.7 & \\
\hline & & Average & 151 & 36.2 & 28 & 41.2 & 179 & 36.9 & \\
\hline & & High & 147 & 35.3 & 20 & 29.4 & 167 & 34.4 & 0.611 \\
\hline \multirow{3}{*}{ Non suicidal } & Type of service & Combat & 299,649 & 40.0 & 157,396 & 32.7 & 457,045 & 37.1 & \\
\hline & & Combat Support & 2,323 & 0.3 & 12,289 & 2.6 & 14,612 & 1.1 & \\
\hline & & Back unit & 446,523 & 59.7 & 312,196 & 64.8 & 758,719 & 61.7 & $<0.001$ \\
\hline \multirow[t]{3}{*}{ Suicide } & Type of service & Combat & 189 & 57.1 & 32 & 47.1 & 221 & 55.4 & \\
\hline & & Combat support & 13 & 3.9 & 14 & 20.6 & 27 & 6.8 & \\
\hline & & Back unit & 129 & 39.0 & 22 & 32.4 & 151 & 37.8 & $<0.001$ \\
\hline \multirow[t]{2}{*}{ Non suicidal } & ${ }^{\mu}$ Psychiatric Diagnosis & No & 971,445 & 99.1 & 473,361 & 98.2 & $1,444,806$ & 98.8 & \\
\hline & & Yes & 9,061 & 0.9 & 8,524 & 1.8 & 17,585 & 1.2 & $<0.001$ \\
\hline \multirow[t]{2}{*}{ Suicide } & ${ }^{\mu}$ Psychiatric Diagnosis & No & 416 & 98.3 & 64 & 94.1 & 480 & 97.8 & \\
\hline & & Yes & 7 & 1.7 & 4 & 5.9 & 11 & 2.2 & 0.029 \\
\hline \multirow[t]{7}{*}{ Non suicidal } & Origin Country & Australia-New Zealand & 7,377 & 0.8 & 2,500 & 0.5 & 9,877 & 0.7 & \\
\hline & & Western country & 2,538 & 26.0 & 82,942 & 17.3 & 336,767 & 23.2 & \\
\hline & & Asia+ Oceania & 219,475 & 22.5 & 81,308 & 17.0 & 300,783 & 20.7 & \\
\hline & & Africa & 220,979 & 22.7 & 91,477 & 19.1 & 312,456 & 21.5 & \\
\hline & & Ethiopia & 17,614 & 1.8 & 17,253 & 3.6 & 34,867 & 2.4 & \\
\hline & & Former Soviet Union & 176,134 & 18.1 & 79,896 & 16.7 & 256,030 & 17.6 & \\
\hline & & Israel & 79,715 & 8.2 & 123,816 & 25.8 & 20,3531 & 14.0 & $<0.001$ \\
\hline \multirow[t]{7}{*}{ Suicide } & Origin Country & Australia-New Zealand & 3 & 0.7 & 2 & 2.9 & 5 & 1.0 & \\
\hline & & Western country & 109 & 26.0 & 13 & 19.1 & 122 & 25.0 & \\
\hline & & Asia+ Oceania & 76 & 18.1 & 10 & 14.7 & 86 & 17.6 & \\
\hline & & Africa & 47 & 11.2 & 10 & 14.7 & 57 & 11.7 & \\
\hline & & Ethiopia & 26 & 6.2 & 7 & 10.3 & 33 & 6.8 & \\
\hline & & Former Soviet Union & 103 & 24.5 & 16 & 23.5 & 119 & 24.4 & \\
\hline & & Israel & 56 & 13.3 & 10 & 14.7 & 66 & 13.5 & 0.371 \\
\hline \multirow[t]{3}{*}{ Non suicidal } & Religion & Other & 5,658 & 0.6 & 36 & 0.0 & 5,694 & 0.4 & \\
\hline & & Druse & 16,496 & 1.7 & 10,051 & 2.1 & 26,547 & 1.8 & \\
\hline & & Jewish & 957,888 & 97.7 & 471,010 & 97.9 & 142,898 & 97.8 & $<0.001$ \\
\hline \multirow[t]{3}{*}{ Suicide } & & Other & 4 & 0.9 & 0 & 0.0 & 4 & 0.8 & \\
\hline & & Druse & 31 & 7.3 & 1 & 1.5 & 32 & 6.5 & \\
\hline & & Jewish & 388 & 91.7 & 67 & 98.5 & 455 & 92.7 & 0.134 \\
\hline \multirow[t]{3}{*}{ Non suicidal } & ${ }^{\sharp}$ SES & Low & 27,336 & 2.9 & 17,019 & 3.6 & 44,355 & 3.1 & \\
\hline & & Average & 479,902 & 50.3 & 245,710 & 51.3 & 725,612 & 50.6 & \\
\hline & & High & 447,666 & 46.9 & 216,143 & 45.1 & 663,809 & 46.3 & $<0.001$ \\
\hline \multirow[t]{3}{*}{ Suicide } & ${ }^{\sharp}$ SES & Low & 22 & 5.4 & 4 & 5.9 & 26 & 5.5 & \\
\hline & & Average & 213 & 52.6 & 39 & 57.4 & 252 & 53.3 & \\
\hline & & High & 170 & 42.0 & 25 & 36.8 & 195 & 41.2 & 0.722 \\
\hline
\end{tabular}

Note: " Before: 1992-2008; ${ }^{\infty}$ After: 2009-2016; ${ }^{\circledR}$ SES-Socioeconomic status. 
Table 3

Univariate analysis with a minimal model adjusted for gender and IQ.

\begin{tabular}{|c|c|c|c|c|c|c|}
\hline \multirow[t]{2}{*}{ Variables } & & \multirow[t]{2}{*}{$\beta$} & \multirow[t]{2}{*}{$p$} & \multirow[t]{2}{*}{$\operatorname{Exp}(\beta)$} & \multicolumn{2}{|c|}{ Univariate 95\% CI } \\
\hline & & & & & LB & UB \\
\hline \multirow[t]{3}{*}{ \#Before } & Intercept & -10.563 & $<0.001$ & 0.00003 & 0.00001 & 0.00005 \\
\hline & Gender & 2.135 & $<0.001$ & 8.459 & 5.983 & 11.958 \\
\hline & Intelligence Score & 0.010 & 0.001 & 1.010 & 1.005 & 1.016 \\
\hline \multirow[t]{3}{*}{${ }^{\infty}$ After } & Intercept & -11.387 & 0.001 & 0.00001 & 0.000002 & 0.00006 \\
\hline & Gender & 1.557 & $<0.001$ & 4.746 & 2.353 & 9.573 \\
\hline & Intelligence Score & 0.012 & 0.090 & 1.012 & 0.998 & 1.027 \\
\hline \multirow[t]{6}{*}{ \#Before } & Intercept & -10.552 & $<0.001$ & 0.00003 & 0.00001 & 0.00006 \\
\hline & Gender & 2.242 & $<0.001$ & 9.413 & 6.072 & 14.592 \\
\hline & Intelligence Score & 0.007 & 0.041 & 1.007 & 1.000 & 1.014 \\
\hline & Combat & 0.408 & $<0.001$ & 1.504 & 1.197 & 1.889 \\
\hline & Combat Support & 3.242 & $<0.001$ & 25.574 & 14.374 & 45.501 \\
\hline & Back unit & $0^{\mathrm{a}}$ & & 1 & & \\
\hline \multirow[t]{6}{*}{${ }^{\infty}$ After } & Intercept & -11.332 & $<0.001$ & 0.00001 & 0.000002 & 0.00007 \\
\hline & Gender & 1.260 & 0.001 & 3.524 & 1.722 & 7.210 \\
\hline & Intelligence Score & 0.009 & 0.234 & 1.009 & 0.994 & 1.024 \\
\hline & Combat & 0.788 & 0.005 & 2.200 & 1.266 & 3.822 \\
\hline & Combat support & 2.450 & $<0.001$ & 11.586 & 5.852 & 22.936 \\
\hline & Back unit & $0^{\mathrm{a}}$ & & 1 & & \\
\hline \multirow[t]{4}{*}{ \#Before } & Intercept & -10.681 & $<0.001$ & 0.00002 & 0.00001 & 0.00005 \\
\hline & Gender & 2.100 & $<0.001$ & 8.167 & 5.772 & 11.556 \\
\hline & Intelligence Score & 0.011 & $<0.001$ & 1.011 & 1.005 & 1.017 \\
\hline & ${ }^{\mu}$ Psychiatric Diagnosis & 0.640 & 0.001 & 1.896 & 1.296 & 2.772 \\
\hline${ }^{\infty}$ After & Intercept & -10.101 & $<0.001$ & 0.0000041 & 0.000002 & 0.00006 \\
\hline & Gender & 1.547 & $<0.001$ & 4.699 & 2.329 & 9.482 \\
\hline & Intelligence Score & 0.012 & 0.092 & 1.012 & 0.998 & 1.027 \\
\hline & ${ }^{\mu}$ Psychiatric Diagnosis & 1.131 & 0.028 & 3.100 & 1.128 & 8.524 \\
\hline${ }^{\#}$ Before & Intercept & -10.101 & $<0.001$ & 0.00004 & 0.00001 & 0.00009 \\
\hline & Gender & 2.092 & $<0.001$ & 8.105 & 5.731 & 11.463 \\
\hline & Intelligence Score & 0.011 & 0.001 & 1.011 & 1.004 & 1.017 \\
\hline & Australia-New Zealand & -0.939 & 0.192 & 0.391 & 0.095 & 1.603 \\
\hline & Western country & -0.460 & 0.006 & 0.631 & 0.456 & 0.874 \\
\hline & Asia + Oceania & -0.593 & 0.001 & 0.552 & 0.391 & 0.781 \\
\hline & Africa & -1.072 & $<0.001$ & 0.342 & 0.232 & 0.505 \\
\hline & Ethiopia & 0.778 & 0.002 & 2.176 & 1.341 & 3. 531 \\
\hline & Former Soviet Union & -0.212 & 0.206 & 0.809 & 0.582 & 1.124 \\
\hline & Israel & 0a & & 1 & & \\
\hline${ }^{\infty}$ After & Intercept & -12.576 & $<0.001$ & 0.000004 & 0.0000005 & 0.00002 \\
\hline & Gender & 1.555 & $<0.001$ & 4.733 & 2.345 & 9.553 \\
\hline & Intelligence Score & 0.017 & 0.025 & 1.018 & 1.002 & 1.033 \\
\hline & Australia-New Zealand & 2.381 & 0.002 & 10.819 & 2.368 & 49.418 \\
\hline & Western country & 0.614 & 0.146 & 1.848 & 0.808 & 4.229 \\
\hline & Asia + Oceania & 0.523 & 0.244 & 1.687 & 0.700 & 4.063 \\
\hline & Africa & 0.438 & 0.330 & 1.550 & 0.642 & 3.743 \\
\hline & Ethiopia & 1.869 & $<0.001$ & 6.479 & 2.361 & 17.780 \\
\hline & Former Soviet Union & 1.018 & 0.012 & 2.768 & 1.254 & 6.110 \\
\hline & Israel & $0 a$ & & 1 & & \\
\hline${ }^{\#}$ Before & Intercept & -10.307 & $<0.001$ & 0.00003 & 0.00001 & 0.00012 \\
\hline & Gender & 2.075 & $<0.001$ & 7.968 & 5.629 & 11.278 \\
\hline & Intelligence Score & 0.012 & $<0.001$ & 1.021 & 1.006 & 1.018 \\
\hline & Other religion & 0.475 & 0.345 & 1.608 & 0.600 & 4.311 \\
\hline & Druse & 1.232 & $<0.001$ & 3.426 & 2.361 & 4.973 \\
\hline & Jewish & $0 \mathrm{a}$ & & 1 & & \\
\hline${ }^{\infty}$ After & Intercept & -11.345 & $<0.001$ & 0.000012 & 0.00000021 & 0.0000066 \\
\hline & Gender & 1.572 & $<0.001$ & 4.818 & 2.389 & 9.728 \\
\hline & Intelligence Score & 0.012 & 0.101 & 1.012 & 0.998 & 1.027 \\
\hline & Other religion & -13.948 & 0.999 & 0.0000009 & 0.000000 & 0.000000 \\
\hline & Druse & -0.650 & 0.520 & 0.522 & 0.072 & 3.784 \\
\hline & Jewish & $0 \mathrm{a}$ & & 1 & & \\
\hline${ }^{\#}$ Before & Intercept & -10.240 & $<0.001$ & 0.0000035 & 0.0000015 & 0.0000082 \\
\hline & Gender & 2.069 & $<0.001$ & 7.915 & 5.591 & 11.204 \\
\hline & Intelligence Score & 0.012 & $<0.001$ & 1.012 & 1.006 & 1.018 \\
\hline & Low SES & -0.531 & 0.020 & 0.588 & 0.375 & 0.921 \\
\hline & Average SES & -0.408 & 0.070 & 0.665 & 0.428 & 1.033 \\
\hline & High SES & $0 \mathrm{a}$ & & 1 & & \\
\hline${ }^{\infty}$ After & Intercept & -11.249 & $<0.001$ & 0.0000013 & 0.00000018 & 0.0000019 \\
\hline & Gender & 1.522 & $<0.001$ & 4.583 & 2.266 & 9.269 \\
\hline & Intelligence Score & 0.015 & 0.050 & 1.015 & 1.000 & 1.030 \\
\hline & Low SES & -0.556 & 0.309 & 0.573 & 0.196 & 1.674 \\
\hline & Average SES & -0.223 & 0.672 & 0.800 & 0.285 & 2.249 \\
\hline & High SES & $0 \mathrm{a}$ & & 1 & & \\
\hline
\end{tabular}

Note: Logistic regression; " Before: 1992-2008; ${ }^{\infty}$ After: 2009-2016; ${ }^{\pi}$ Psychiatric Diagnosis in recruit day. 
declined after the SPP from an OR of 25.574 (95\% CI, 14.374-45.501; $p<0.001)$ to $11.586(95 \% \mathrm{CI}, 5.852-22.936 ; p<0.001)$. Meanwhile for combat soldiers, the OR increased after the SPP $(\mathrm{OR}=1.504 ; 95 \%$ $C I, 1.197-1.889 ; p>0.001$ and $\mathrm{OR}=2.200 ; 95 \% \mathrm{CI}, 1.266-3.822$; $p=0.005$ ).

The absolute number of soldiers with psychiatric diagnosis who DBS in the years following the SPP was rather small in comparison to the absolute number from the years before. However, the overall DBS risk (as was shown in absolute numbers) for the entire soldier population declined more than the risk for soldiers with psychiatric illness. As a result, while statistically the risk for DBS among soldiers with psychiatric diagnosis before the implementation of the program was slightly elevated $(\mathrm{OR}=1.896 ; 95 \% \mathrm{CI}, 1.296-2.772 ; p=0.001)$, the risk after was found to have greatly increased (OR=3.100; 95\% CI, 1.128 8.524; $p=0.028$ ).

As can be seen in Table 3, when Israeli ethnicity was used as point of reference, the risk for DBS before the SPP was highest among soldiers from Ethiopian ethnicity $(\mathrm{OR}=2.176$; $95 \% \mathrm{CI}, 1.341$ $3.531 ; p=0.002$ ) but other ethnicities actually served as protecting factors. After the implementation of the SPP, the risk for DBS rose for all soldiers of non-Israeli ethnicity, but the two populations with the highest risk proved to be soldiers of Ethiopian ethnicity for whom the OR is $6.479(95 \% \mathrm{CI}, 2.361-17.780 ; p<0.001)$ and soldiers from Australia-New Zealand ethnicity (OR=10.819; $95 \% \mathrm{CI}$, 2.368-49.418; $p=0.002$ ).

Another interesting finding presented in Table 3 regards religious minority. Before the SPP, being part of a religious minority, mainly Druse (OR=3.426; 95\% CI, 2.361-4.973; $p<0.001$ ), constituted a risk factor for DBS (frequency and percentages of DBS among Druse soldiers presented in Table 2), but it became a protective factor after SPP.

\subsection{Results summary}

The analysis revealed that before the SPP implementation, those of male gender (OR, 7.885; 95\% CI, 5.071-12.259; $p<0.001)$, high intelligence score (OR, 1.012; 95\% CI, 1.004-1.019; $p=0.002)$, having a psychiatric diagnosis (OR, 2.422; 95\% CI, 1.526-3.842; $p<0.001$ ), being in a combat support unit (OR, $14.962 ; 95 \% \mathrm{CI}$, 8.427-26.563; $p<0.001$ ), being of Druse religion (OR, 4.027; 95\% CI, 2.211-7.331; $p<0.001)$ and being of Ethiopian ethnicity (OR, 3.522; 95\% CI, 1.2891-6.650; $p<0.001$ ), were at increased risk for DBS. After the implementation of SPP, male gender still constituted a higher risk (OR, 3.281; 95\% CI, 1.600-6.726; $p=0.001$ ), but the risk level decreased from $\mathrm{OR}=7.885$ before to $\mathrm{OR}=3.281$ after SPP. Other risk factors still included high intelligence score (OR, 1.017; $95 \% \mathrm{CI}, 0.001-1.033 ; p=0.037)$ and having a psychiatric diagnosis on recruitment day (OR, 5.830; 95\% CI, 2.046-16.612; $p=0.001$ ) were the $\mathrm{OR}=2.422$ before SPP changed to $\mathrm{OR}=5.830$ after SPP. Also serving in a combat support unit (OR, 6.304; 95\% CI, 3.334-11.919; $p<0.001)$ and being of non-Israeli ethnicity and especially Ethiopian (OR, 8.130; 95\% CI, 2.868-23.047; $p<0.001$ ) presented risk factors for DBS.

\section{Discussion}

Over the last few years, it has become clear that the SPP implemented in the IDF succeeded in lowering rates of DBS in the Israeli army (Fig. 1). This was demonstrated in a number of publications $[3,4]$, as well as in the current study.

Despite it being successful in improving the situation significantly, the SPP does not prevent all DBSs. It seems that more research is necessary in order to modify the SPP so that it helps minimize the number of DBS's among soldiers in active military service. The soldiers in the IDF, whose conscription is mandated by law, represent a sub-cohort of Israeli society, thus resulting in a highly heterogeneous population. The current study attempted to compare characteristics of soldiers who died by suicide prior to the SPP implementation (2009-2016), with characteristics of soldiers who committed DBS after SPP implementation (1992-2008). The research question was whether changes would be found and if so, the nature of those changes. Before implementing the SPP, risk factors for suicide were both dispositional (gender, cognitive level, socio-economic status, mental illness etc.) and situational (serving in a combat unit). The assumption was that after the SPP and reducing the rate of suicide, dispositional factors would be more dominant. This assumption was based on an earlier theory, which held that the contribution of dispositional and situational factors to suicide among combat soldiers may differ from the contribution to suicide among non-combatants [10].

The current research, shows a larger change in the situational factors of soldiers who died by suicide after the SPP implementation than in their dispositional ones.

The study found that male soldiers, were affected more than female soldiers by the SPP, though still maintaining their status as a high-risk population for DBS. One of the main SPP strategies was restriction of fire-arms [12]. Since male soldiers constitute the majority of the general military population and especially the vast majority of combat soldiers, that strategy has a higher impact on the male population. Thus, despite male gender being a dispositional risk factor for DBS it acts like a situational one. In a few years, we may see similar changes in the female population as well since more military combat professions opened up for women in recent years.

Service in a combat support unit was found to be a stronger risk factor compared to service as a combat soldier or back unit soldier. Combat support soldiers, while serving in a stressful environment with routine access to fire-arms, identical to that of combat soldiers, tend to be less physically and mentally fit, less motivated and more likely to suffer from adjustment difficulties. The current study shows that the SPP targeted this population successfully, probably by reducing their access to fire-arms, thus reducing by half their risk for DBS. Still, they remain a very high-risk population.

Another finding was the rise in the proportion of soldiers with known mental health disorders among the total number of DBSs. This suggests that the SPP was more effective approaching healthy soldiers facing situational factors, while dispositional factors, such as mental disorders, were tougher to approach. The IDF's Mental Health Department has taken several steps in order to improve accessibility of Mental Health Officers for the soldiers. It also instituted a follow-up plan to examine wheatear that better-access policy indeed leads to a decrease in DBS among soldiers with mental health disorder.

An additional finding was a decline after SPP in the average intelligence score of those who committed DBS. This change, however, was not statistically significant and high intelligence remains a risk factor for DBS.

Socioeconomic status was shown to have no relevance as a risk factor, both before and after the SPP. The authors though believe that the socioeconomic index used in the IDF is not sensitive enough since it represents the socioeconomic status only at settlement cluster level rather than at individual family level.

Before the SPP, the only ethnicity that was found significant as risk for DBS was Ethiopian (Table 4). After the SPP, overall suicidality declined, however, Ethiopian ethnicity remained a relative risk factor and even increased in the proportional risk it represents. Other ethnicities became a relative risk factor as well. Here it should be noted again that the authors believe that being an immigrant serves as a dispositional risk factor, rather than a situational one and is thus impacted less by the SPP. This issue 
Table 4

Multivariate analysis with suicide binary logistic dependent variable before and after implementation of the SPP.

\begin{tabular}{|c|c|c|c|c|c|}
\hline \multirow[t]{2}{*}{ Variables } & \multirow[t]{2}{*}{$\beta$} & \multirow[t]{2}{*}{$p$} & \multirow{2}{*}{$\begin{array}{l}\operatorname{Exp}(\beta) \\
\text { OR }\end{array}$} & \multicolumn{2}{|c|}{ univariate $95 \% \mathrm{CI}$} \\
\hline & & & & LB & UB \\
\hline${ }^{\#}$ Before Intercept & -10.769 & $<0.001$ & 0.000002 & 0.0000002 & 0.000006 \\
\hline Gender & 2.065 & $<0.001$ & 7.885 & 5.071 & 12.259 \\
\hline Intelligence Score & 0.012 & 0.002 & 1.012 & 1.004 & 1.019 \\
\hline Psychiatric diagnosis & 0.884 & $<0.001$ & 2.422 & 1.526 & 3.842 \\
\hline Combat support & 2.705 & $<0.001$ & 14.962 & 8.427 & 26.563 \\
\hline Back unit & -0.448 & $<0.001$ & 0.639 & 0.505 & 0.808 \\
\hline Combat & $0^{\mathrm{a}}$ & & 1 & & \\
\hline Australia-New Zealand & -0.066 & 0.928 & 0.936 & 0.221 & 3.971 \\
\hline Western country & 0.049 & 0.838 & 1.050 & 0.658 & 1.675 \\
\hline Asia + Oceania & -0.077 & 0.758 & 1.926 & 0.569 & 1.507 \\
\hline Africa & -0.608 & 0.028 & 0.554 & 0.317 & 0.935 \\
\hline Ethiopia & 1.259 & $<0.001$ & 3.522 & 1.891 & 6.560 \\
\hline Former Soviet Union & 0.286 & 0.235 & 1.331 & 0.830 & 2.132 \\
\hline Israel & $0^{\mathrm{a}}$ & & 1 & & \\
\hline Atheist & 0.347 & 0.498 & 1.414 & 0.519 & 3.855 \\
\hline Druse & 1.393 & $<0.001$ & 4.027 & 2.211 & 7.331 \\
\hline Jewish & $0^{\mathrm{a}}$ & & 1 & & \\
\hline High SES & 0.123 & 0.686 & 1.131 & 0.624 & 2.051 \\
\hline Average SES & 0.192 & 0.508 & 1.212 & 0.686 & 2.140 \\
\hline Low SES & oa & & 1 & & \\
\hline${ }^{\infty}$ After Intercept & -11.371 & $<0.001$ & 0.000001 & 0.0000001 & 0.000000 \\
\hline Gender & 0.188 & 0.001 & 3.281 & 1.600 & 6.726 \\
\hline Intelligence Score & 0.017 & 0.037 & 1.017 & 0.001 & 1.033 \\
\hline Psychiatric diagnosis & 1.763 & 0.001 & 5.830 & 2.046 & 16.612 \\
\hline Combat support & 1.841 & $<0.001$ & 6.304 & 3.334 & 11.919 \\
\hline Back unit & -0.901 & 0.002 & 0.406 & 0.231 & 0.716 \\
\hline Combat & $0^{\mathrm{a}}$ & & 1 & & \\
\hline Others & 2.551 & 0.001 & 12.815 & 2.765 & 59.400 \\
\hline Western country & 0.746 & 0.086 & 2.109 & 0.900 & 4.942 \\
\hline Asia + Oceania & 0.705 & 0.126 & 2.023 & 0.821 & 4.983 \\
\hline Africa & 0.574 & 0.217 & 1.776 & 0.714 & 4.417 \\
\hline Ethiopia & 2.096 & $<0.001$ & 8.130 & 2.868 & 23.047 \\
\hline Former Soviet Union & 1.228 & 0.003 & 3.415 & 1.499 & 7.780 \\
\hline Israel & $0^{\mathrm{a}}$ & & & & \\
\hline Atheist & -14.099 & 0.999 & 0.00000 & 0.0000 & 0.0000 \\
\hline Druse & -0.337 & 0.762 & 0.714 & 0.080 & 6.340 \\
\hline Jewish & $0^{\mathrm{a}}$ & & 1 & & \\
\hline High SES & -0.975 & 0.093 & 0.377 & 0.121 & 1.175 \\
\hline Average SES & -0.674 & 0.230 & 0.510 & 0.170 & 1.5329 \\
\hline Low SES & $0 a$ & & 1 & & \\
\hline
\end{tabular}

Note: " Before: 1992-2008; ${ }^{\infty}$ After:2009-2016.

warrants further research at a future time, with larger cohorts of soldiers who died by suicide.

The risk factor of belonging to a religious minority changed too. While before the SPP being a Druse soldier constituted a risk factor for DBS, after the SPP this risk declined substantially and became a protective factor. This finding may result from the SPP itself or from an ongoing trend in the IDF in the last decade, to educate commanders and health professionals (including mental health officers) in diversity and how to approach other populations. The SPP having increased mental health accessibility especially in field units where most Druse soldiers serve, may also account for this finding [13]. It should still be taken with caution, since the absolute number of Druse suicides was relatively low both before $(n=31$, $7.3 \%)$ and after $(\mathrm{n}=1,1.5 \%)$ SPP (Table 2$)$.

\section{Conclusion}

Current risk factors for suicide in the IDF post-SPP implementation includes male gender, serving in a support unit, high intelligence score, psychiatric illness as well as Ethiopian or other non-Israeli ethnicity. The SPP has proven successful over the years in reducing the rate of DBS among IDF personnel. Our study shows that the SPP approached differently various risk factors, and was found to be more effective regarding situational factors than regarding dispositional ones. Thus dispositional factors became more dominant in the population that committed DBS after SPP implementation.

The only dispositional factor that acted differently (found to be less dominant) was male gender. The authors believe that this is due to males constituting the vast majority of combat soldiers and thus having more access to fire-arms - a serious risk factor for DBS.

More research is required to find additional ways to further lower the rate of DBS in the IDF and to modify the SPP accordingly.

\section{Limitations}

In 2008, two years after starting the SPP and in the middle of its implementation, there is $d$ is very remarkable. The fact that it's a transition time makes distinguishing the affect more problematic. Thus, scientifically speaking, it makes much more sense to ignore data from 2007 to 2009. That makes this study's findings more conservative, as should be.

One should keep in mind that the number of DBS for which data was available, is relatively small (491), especially as a percentage of the whole soldier population which numbers 472,795 soldiers. One should also note that including all significant variables from the univariate analysis into the multivariate analysis causes a reduction in the number of available cases for the analysis, in 
such manner that the benefit of adding the independent variables to the Multivariate model (after having narrowed down variables with similar meanings and collinearity) is less than the damage in the power of the statistical funding heating the capacity to the entire population.

\section{References}

[1] Tzur D, Kedem R, Twig G, Ben Yehuda A, Abergil M, Glassberg E. Death circumstances in IDF: description data for 1990-2016. J Israeli Mil Med Special Ed Mental Health Care IDF 2018;15(39-40)50-6 2-3.

[2] Shelef L, Laur L, Raviv G, Fruchter E. A military suicide prevention program in the Israeli Defense Force: a review of an important military medical procedure. Disaster Mil Med 2015;1(1):16, doi:http://dx.doi.org/10.1186/s40696-0150007-y.

[3] Shelef L, Laur L, Derazne E, Mann JJ, Fruchter E. An effective suicide prevention program in the Israeli Defense Forces: a cohort study. Eur Psychiatry 2016;31:37-43, doi:http://dx.doi.org/10.1016/j.eurpsy.2015.10.004.

[4] Shelef L, Tomer G, Tatsa-Laur L, Kedem R, Bonne O, Fruchter E. Risk factors for suicide in the Israeli army between the years 1992-2012: a case-control study. Eur Psychiatry 2017;39:106-13, doi:http://dx.doi.org/10.1016/j. eurpsy.2016.08.005.

[5] Griffith J, Bryan CJ. Suicides in the U.S. military: birth cohort vulnerability and the all-volunteer force. Armed Forces Soc 2015;1-18, doi:http://dx.doi.org/ $10.1177 / 0095327 \times 15614552$.
[6] Gilman SE, Bromet EJ, Cox KL, Colpe LJ, Fullerton CS, Gruber MJ, et al. Sociodemographic and career history predictors of suicide mortality in the United States Army 2004-2009. Psychol Med 2014;44:2579-92, doi:http://dx. doi.org/10.1017/S003329171400018X.

[7] Pruitt L, Smolenski D, Reger M, Bush N, Skopp N, Campise R. Department of defense suicide event report calendar year 2014 annual report. Distributed by the National Center for Telehealth \& Technology (T2) \& Defense Centers of Excellence for Psychological Health \& Traumatic Brain Injury (DCoE). 2016.

[8] Bachynski KE, Canham-Chervak M, Black SA, Dada EO, Millikan AM, Jones BH. Mental health risk factors for suicides in the US Army, 2007-8. Inj Prev 2012;18 (6):405-12, doi:http://dx.doi.org/10.1136/injuryprev-2011-040112.

[9] Zheng Yingye. Evaluating prognostic accuracy of biomarkers in nested casecontrol studies". Biostatistics 2012;13(1):89-100, doi:http://dx.doi.org/ 10.1093/biostatistics/kxr021.

[10] Bodner E, Ben-Artzi E, Kaplan Z. Soldiers who kill themselves: the contribution of dispositional and situational factors. Arch Suicide Res 2006;10:29-43, doi: http://dx.doi.org/10.1080/13811110500318299.

[11] Twig G, Gluzman I, Tirosh A, Gerstein HC, Yaniv G, Afek A, et al. Cognitive function and the risk for diabetes among young men. Diabetes Care 2014;37:2982-8, doi:http://dx.doi.org/10.2337/dc14-0715.

[12] Lubin G, Werbeloff N, Halperin D, Shmushkevitch M, Weiser M, Knobler HY. Decrease in suicide rates after a change of policy reducing access to firearms in adolescents: a naturalistic epidemiological study. Suicide Life Threat Behav 2010;40(5):421-4, doi:http://dx.doi.org/10.1521/suli.2010.40.5.421.

[13] Shelef L, Essami N, Birani A, Hartal M, Yavnai N. Personal and psychiatric characteristics among Druze soldiers attempting suicide during military service. J Affect Disord 2019, doi:http://dx.doi.org/10.1016/j.jad.2019.06.011. 